\title{
Porous titanium for biomedical applications: An experimental study on rabbits
}

\author{
Luana-Marotta-Reis de Vasconcellos ${ }^{1}$, Daniel-de Oliveira Leite ${ }^{1}$, Fernanda-Oliveira Nascimento ${ }^{1}$, Luis- \\ Gustavo-Oliveira de Vasconcellos ${ }^{3}$, Mário-Lima-de Alencastro Graça ${ }^{2}$, Yasmin-Rodarte Carvalho ${ }^{1}$, Carlos- \\ Alberto-Alves Cairo ${ }^{2}$
}

\author{
${ }^{1}$ Department of Bioscience and Buccal Diagnosis, São Jose dos Campos School of Dentistry, State University of São Paulo \\ (UNESP), São José dos Campos, Brazil \\ ${ }^{2}$ Department of Materials, Air and Space Institute, CTA, São José dos Campos, Brazil \\ ${ }^{3}$ Department of Prostodontics and Dental Materials, São Jose dos Campos School of Dentistry, State University of São Paulo \\ (UNESP), São José dos Campos, Brazil
}

Correspondence:

Alameda Harvey C. Weeks, 14, sl. 09

Vista Verde, São José dos Campos

12223-830, SP, Brazil

luanamrv@itelefonica.com.br

\begin{abstract}
Vasconcellos LM, Leite DO, Nascimento FO, Vasconcellos LG, Graça ML, Carvalho YR, Cairo CA. Porous titanium for biomedical applications: An experimental study on rabbits. Med Oral Patol Oral Cir Bucal. 2010 Mar 1;15 (2):e407-12.

http://www.medicinaoral.com/medoralfree01/v15i2/medoralv15i2p407.pdf
\end{abstract}

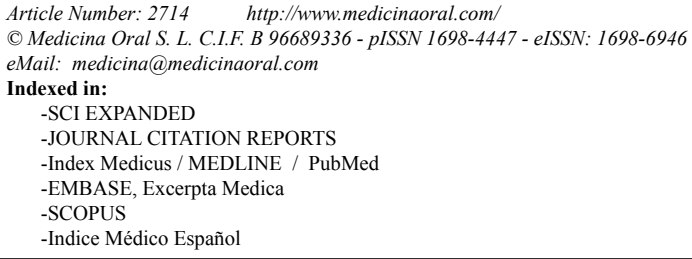

\begin{abstract}
Objective: The aim of this study was to carry out an in vivo assessment of bone ingrowth in two different types of porous titanium -the first being completely porous, and the second with a porous surface and dense nucleus, manufactured by powder metallurgy- and to evaluate their mechanical properties. Study design: Ten scaffolds from each group were submitted to metallographic analysis and compression tests. Next, two scaffolds of each type were inserted into 14 rabbits, which were sacrificed 8 weeks after surgery. The samples were submitted for histological examination. Results: Metallographic analysis revealed interconnected pores, and the average interconnected pore diameter was about $360 \mathrm{~mm}$, with $36 \%$ total porosity. The totally porous titanium samples and the titanium samples with porous surface and dense nucleus showed an average compressive strength of $16.19 \mathrm{MPa}$ and $69.27 \mathrm{MPa}$, respectively. After 8 weeks, the animals showed bone ingrowth, even into the most internal pores. Conclusions: The pore morphology was effective in permitting bone ingrowth in both groups. Titanium scaffolds with a porous surface and dense nucleus showed the best mechanical properties and most adequate interface.
\end{abstract}

Key words: Porosity, osseointegration, titanium, mechanical properties, biomedical. 


\section{Introduction}

Titanium is widely used for the production of dental or orthopedic implants because direct contact occurs between bones and implant surfaces (1). Titanium is biocompatible, highly corrosion resistant and durable. Moreover, it is easily prepared in many different shapes and textures without affecting its biocompatibility (2).

However, most titanium implants consist of dense components, which lead to problems such as bone resorption and implant loosening due to biomechanical mismatch of the elastic modulus (3). To overcome these problems, porous structures are being investigated extensively, since a reduction in elastic modulus can be coupled with bone integration through tissue ingrowth into pores (4). The interaction between mechanical signals and biological processes in cells and tissue is studied in mechanobiology. Mechanical load may influence cell proliferation, differentiation and metabolism and therefore have a crucial role in live tissue growth, adaptation, regeneration and bioengineering (5).

Bone ingrowth into the porous structure of the implant provides a strong implant/bone bond (6), and these pores can be interconnected three-dimensionally. The interconnected pores provide enough space for the attachment and proliferation of new bone tissues and facilitate the transport of body fluids (7). Several factors are important for promoting cell growth, such as pore shape and size, as well as their interconnectivity and spatial distribution throughout the scaffold. Scaffold architecture is crucial for allowing vascularization and the supply of nutrients to the developing tissue. Studies have shown that the optimum pore size required for scaffolds fixation remains undefined, the consensus is that in order to optimize mineralized bone ingrowth, pore sizes between 100 and $500 \mu \mathrm{m}$ are required (6-8).

These porous structures have many applications ran-ging from spinal fixation to acetabular hip prostheses, dental implants, permanent osteosynthesis plates, and intervertebral discs (9). In general, porous-surfaced Ti-based scaffolds can be manufactured by one of the following techniques: plasma-spraying (9), anodic dissolution, and grit blasting (10), but these techniques produce only cavities and not interconnected pores. However, there are few efficient techniques for manufacturing these complex shapes with interconnected pores without the need for machining steps (11) such as powder metallurgy (PM) (6-8), the multiple coating technique (12), and powder sintering techniques (13). The powder metallurgy technique seems to be particularly advantageous because of its processing route and cost (11). In powder metallurgy, pores can originate from the particle compacting arrangement or from changes in this arrangement, when decomposition of spacer particles causes increasing porosity, and from solid-state diffusion in the sintering step (14).
Finally, the porous structure must also present adequate mechanical strength, since large pores have a deleterious effect on the scaffold's mechanical properties. The gradient of maximum porosity must be adjusted adequately with respect to porosity and pore size, in order to ensure the scaffold's acceptable mechanical strength $(11,15)$.

The objective of the present study was to make an in vivo evaluation of bone ingrowth in two types of porous titanium manufactured by the powder metallurgy technique, and to evaluate their mechanical properties.

\section{Materials and Methods}

Scaffolds materials

Two different types of porous titanium, the first totally porous and the second with a porous surface and dense nucleus, were manufactured by powder metallurgy technique. The materials used to manufacture the scaffolds were commercially pure titanium powder with a mean particle size of about $8 \mu \mathrm{m}$, formed by hydrogenation and dehydrogenation (HDDH) and urea with a particle size of about $200 \mu \mathrm{m}$ used as spacer material. The selection of the size of the urea particles was chosen according to previous investigations by our group, to control the amount and size of the spacer particles (6-8). These particles functioned as spacer material.

Both scaffolds were prepared from a titanium/urea powder mixture, having a ratio of $80 \%$ weight to $20 \%$ weight, respectively. The dense nucleus titanium samples were produced with pores exhibiting adequate distribution, since they were located only on the sample surface. A dense nucleus aimed to optimize the mechanical properties of the samples.

The powders were pressed uniaxially at $100 \mathrm{MPa}$ into a stainless steel mold and then pressed isostatically at 200MPa. The porous cylindrical samples were heattreated at $180^{\circ} \mathrm{C} / 2 \mathrm{~h}$ in air to burn out the spacer particles. Both samples were sintered at $1200^{\circ} \mathrm{C} / 1 \mathrm{~h}$, under vacuum (10-7 torr).

After sintering, the totally porous scaffold had an average diameter of $3.0 \mathrm{~mm}$ and a length of $6.0 \mathrm{~mm}$, and the scaffold with a porous surface and dense nucleus had an average diameter of $4.5 \mathrm{~mm}$ and a length of $6.0 \mathrm{~mm}$ (Fig. 1). Pore size and distribution in the finished scaffold were controlled by the particle size and quantity of urea added to the titanium powder. The evaporation of the urea particles left pores in the metal microstructure, without residues.

Pore size and distribution - metallographic analysis

The scaffolds were embedded in acrylic resin and sectioned longitudinally (Labcut 1010-EXTEC). All the specimens were polished lightly using increasingly fine sandpapers. After the metallographic preparation, the specimens' pore size, distribution and connectivity were analyzed in a scanning electron microscope (LEO $435 \mathrm{VPI})$ with $100 \mathrm{X}$ magnification. The pore percen- 


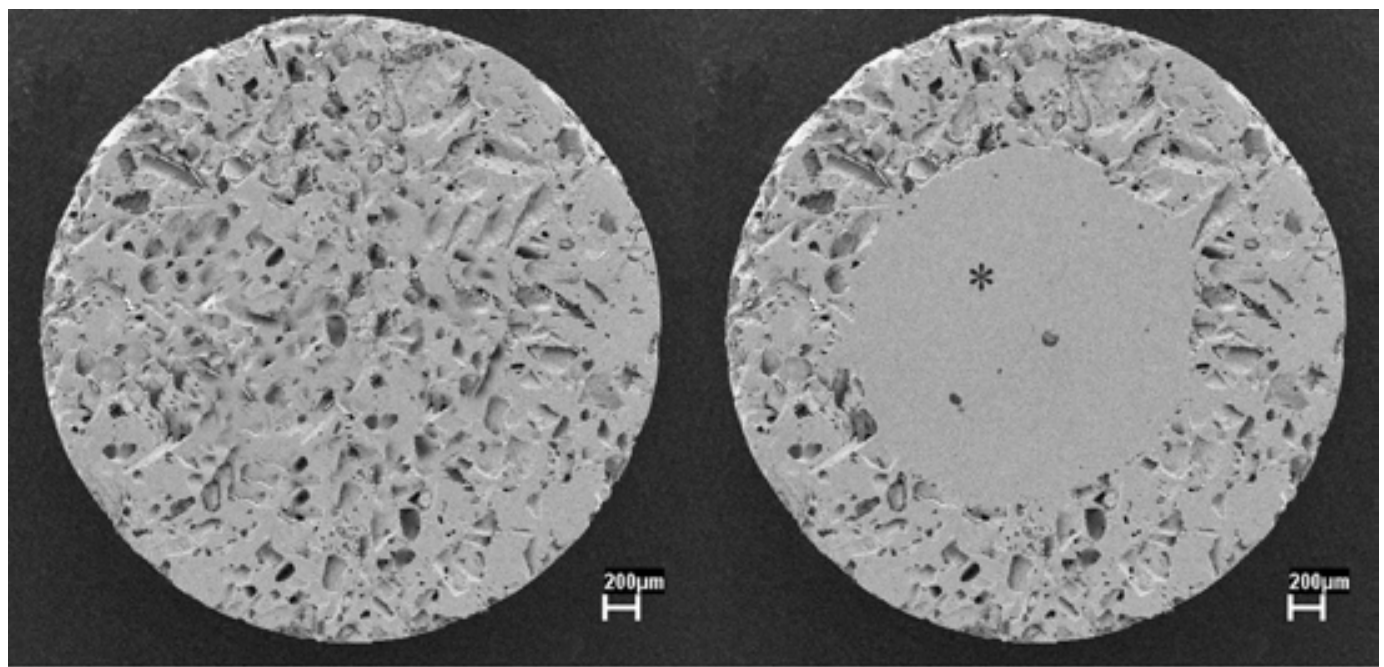

Fig. 1. Cross-section of a scaffold produced by PM: a) totally porous titanium $(*) ;$ b) porous surface $(*)$ and dense nucleus $\left({ }^{*}\right)$.

tage and diameter were analyzed using the Image Tool (Windows 3.00) software program. All data were analyzed by descriptive statistical analysis.

\section{Compression test}

The scaffolds thus developed were subjected to a compression test to evaluate their mechanical properties. Five specimens from each group were tested in uniaxial compression, using a mechanical testing machine (MTS08 machine) at a cross-head speed of $0.5 \mathrm{~mm} / \mathrm{min}$. The test was performed at $37^{\circ} \mathrm{C}$.

It was necessary to determine the area where the force was applied, in order to determine the stress required to break the scaffold. The average height was calculated and used to determine the area according to the following formula: mean area $(\mathrm{Am})=2 \pi \mathrm{r} \times$ average height, where $r=$ scaffold radius. The scaffolds' stress rupture strength was then calculated from the Equation: $\sigma=\mathrm{F} /$ Am, where $\sigma=$ shear stress; $F=$ peak load at failure.

All data were statistically analyzed using two-way parametric ANOVA and the Tukey test, where differences of $\mathrm{p} \leq 0.05$ were considered statistically significant.

Surgical procedure

Fourteen New Zealand albino rabbits, five months old and with a mean weight of $4.0 \mathrm{~kg}$, were used in this study. The animals were supplied by the vivarium of the São José dos Campos School of Dentistry and were kept in individual cages and fed with commercial pet food and water ad libitum. The animals received two totally porous scaffolds in the left tibiae, and two titanium scaffolds with porous surface and dense nucleus in the right tibiae. This study was approved by the Research Ethics Committee, Graduate School of Dentistry of São José dos Campos UNESP (044/2002).

Prior to the surgery, the animals were weighed and anesthetized intramuscularly with a mixture of $13 \mathrm{mg} /$ $\mathrm{kg}$ of an aqueous solution of $2 \%$ hydrochloride, 2-(2,6-
xylidine)-5.6-dihydro-4H-1.3-thiazin (Rompum - Bayer), an analgesic, sedative and muscular relaxant, and with $33 \mathrm{mg} / \mathrm{Kg}$ of ketamine (Dopalen - Agibrands do Brazil Ltda), a general anesthetic. A local anesthetic composed of $3 \%$ octapressin combined with prilocaine hydrochloride and felypressin was also used.

The procedures were performed under the usual standard sterile conditions. After trichotomy, shaving, disinfection, and draping, a straight skin incision of $3 \mathrm{~cm}$ was made in the medial portion of the tibiae. The fascia was split, and the implantation sites were prepared slowly and carefully using an electronic surgical drill. During drilling, the hole was continuously cooled with saline. Just before insertion of the scaffolds, the hole was irrigated with saline to remove any bone shards.

The scaffold was removed from the wrap, placed in the perforation and pressed into the surgical cavity until it was fixed to the cortical bone, after which the tissue was sutured. All the animals were then given the antibiotic penicillin. Eight weeks after implantation the rabbits were sacrificed by applying an overdose of the anesthetic solution intramuscularly.

\section{Results \\ Metallographic analysis}

The metallographic analysis of the porous scaffolds revealed different types of pores, most of them interconnected (Fig. 2) and a few of them isolated. The average interconnected pore diameter was about $360 \mu \mathrm{m}$, with $36 \%$ total porosity.

In the metallographic analysis it not observed the presence of crack between them, suggesting appropriate transition gradient.

Histological analysis

All the animals presented satisfactory postoperative results, with no evidence of inflammation or infection 
in the surgical sites. New bone was observed at the scaffold-bone interface, regardless of the type of scaffold, leading to osseointegration (Fig. 3). Moreover, both types of scaffolds also showed new bone in their pores. This new bone was constituted of mature bone trabeculae with a lamellar arrangement and different sized medullar spaces. There was a distinct edge between newly formed and preexisting bone, emphasizing the biocompatibility of the material and the adequate surface for new bone proliferation

All the animals showed bone ingrowth, even into the most internal pores. New bone was also observed above and below the scaffolds, and the pores of these areas also presented new bone. No fibrous tissue was observed at the bone- scaffold interface.

\section{Compression test}

The totally porous titanium samples and the titanium samples with porous surface and dense nucleus showed an average compressive strength of $16.19 \mathrm{MPa}$ and 69.27MPa, respectively.

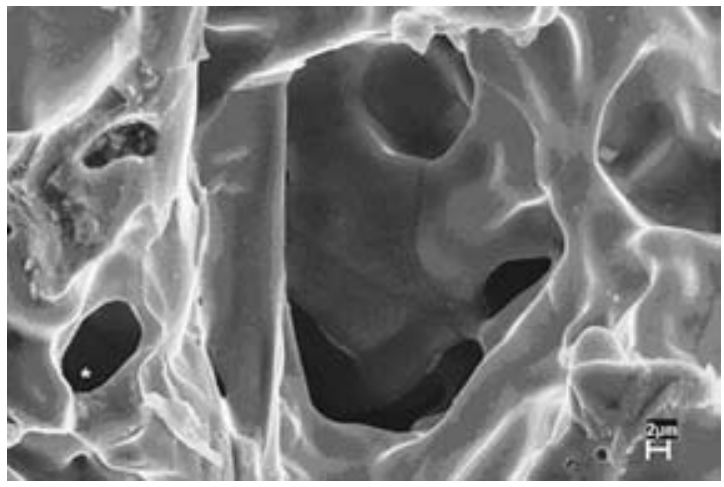

Fig. 2. Porous structure, interconnecting pores (*).

\section{Discussion}

In the present study, two types of titanium scaffolds were manufactured by powder metallurgy techniques, one totally porous or and the other with porous surface and dense nucleus. The pores of both scaffolds allowed for bone ingrowth, even into the innermost pores, evidencing the three-dimensional growth. However, the scaffolds with porous surface and dense nucleus displayed greater compressive strength.

Porous materials can provide early mechanical stabilization, preventing early mobility and micromotion due to their increased surface (6-8). Their capacity for osseointegration also offers the advantage of better longterm fixation, good biofunctionality and biocompatibility (6). Titanium foam presenting porosity not only at the scaffold surface but throughout the entire structure may allow for a higher degree of bone infiltration, while also reducing the stiffness of the foam when compared with that of solid titanium, thereby addressing the stress shielding problem (4). In the present study, the totally porous scaffold showed bone ingrowth inside its pores, but mechanically, this porous metallic structure was relatively weak. An alternative to totally porous Ti were the samples with porous surface and dense nucleus, which also showed bone ingrowth within pores and adequate elastic modulus. From a histological standpoint, the two samples of porous titanium showed a similar amount and quality of bone ingrowth.

The porous surface acts as a gradient with respect to material hardness, where the bone shows greater flexibility and reduced hardness, followed by the porous surface filled with bone and finally the dense titanium, which presents the lowest elasticity and greatest hardness. The porous structure filled with bone is a composite, an important area of transition between the titanium core and original bone. Metals like titanium are
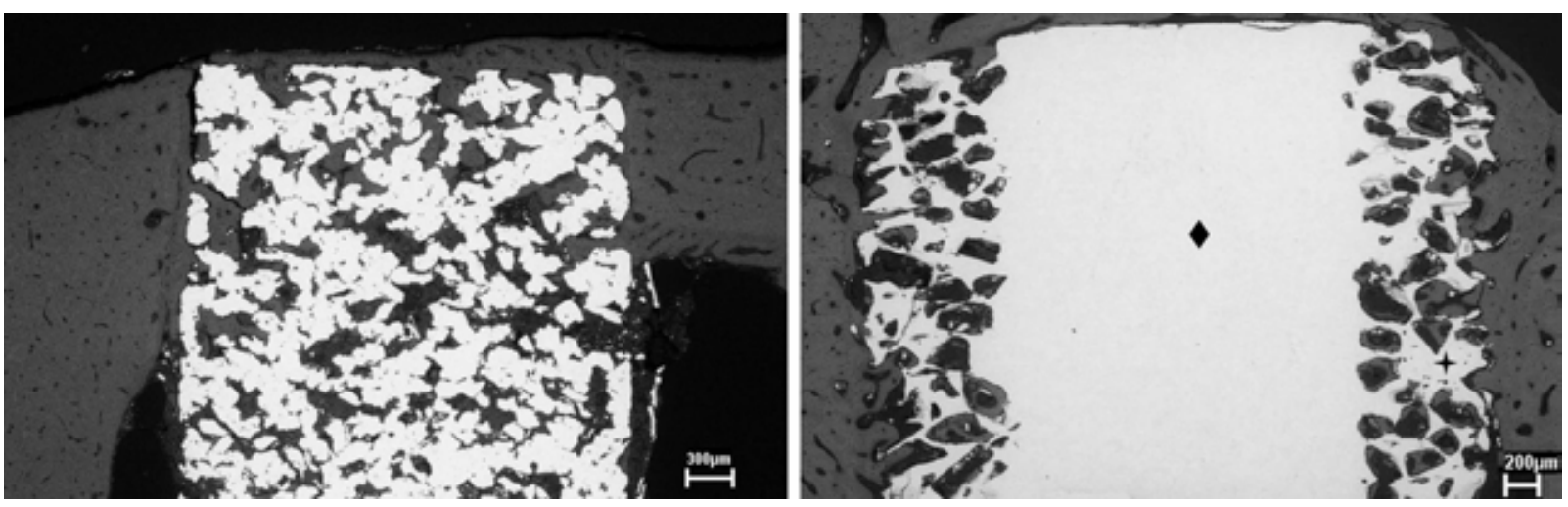

Fig. 3. Osseointegration and new bone was observed growing into the porous surface titanium scaffold, regardless of the type of scaffold: a) porous surface $(*)$ and dense nucleus $(*) ;$ b) totally porous titanium. 
much stiffer than bone and an implant of solid Ti can carry a disproportionate amount of the biological load. The surrounding bone is then stress shielded, which can lead to bone resorption (16). The porous surface titanium offers certain advantages over solid Ti, including enhancing bone-implant interface strength by promoting bone ingrowth and reducing the bone-implant modulus mismatch, which can lead to stress shielding. Therefore, biomaterials must exhibit hardness similar to bone (16), without producing a systemic or localized immune response (17). In addition, the material to be implanted must present mechanical properties adequate for the propose, while also presenting elasticity values similar to bone (17).

The mechanical properties of titanium products made of PM depend on the material's composition, and on the density and final microstructure of the compact. Therefore, specially prepared metal powders of round shapes and sizes of 10-30 $\mu \mathrm{m}$ are required (3). In this study, both scaffolds were produced with titanium powders whose particles were spherical and had a size of $8 \mu \mathrm{m}$, and the final microstructure of the compact was adequate. Thus, the higher compressive strength was due to the presence of the dense nucleus.

Characteristics such as pore diameter and porosity still require investigation. Because of the great difficulties to correlate surface properties with clinical results, the ideal microtopography for commercial porous scaffolds is not yet known (13). The diameter of interconnecting pores seems to dictate the quality of tissue growing into the porous spaces. Although there are alternative views, the consensus seems to be that the optimal pore size for mineralized bone ingrowth is $100-500 \mu \mathrm{m}(6-8)$. The pores of both porous titanium samples had an average diameter of $360 \mu \mathrm{m}$, which sufficed for the consistent ingrowth of new bone within the porous spaces. A suitable porous network is required to promote extensive vascularization for bone ingrowth, rapid bone regeneration and good scaffold integration. Bone ingrowth rates depend to a large extent on pore morphology, the degree of pore connectivity, and pore volume (15), and it is known that pore connectivity is necessary to enable access of blood and nutrients for bone mineralization. On the other hand, larger pore sizes could weaken the porous scaffolds as a result of an associated reduction in density (18).

Dental and orthopedic implants can make use of osteoconductive surfaces such as hydroxyapatite to promote increased implant fixation to bone. There may be failure between this coating and the metal substrate. Thus, porous implants have been studied to promote implant fixation through bone growth into the porous structure and to provide an alternative to osteoconductive surfaces (19). The porous surface and dense nucleus implants developed in this study were produced in a single step; therefore, there was no failure between porous coating and the metal substrate, as sometimes reported for others surfaces. The porous surface might be an alternative to dental implant in less favorable conditions (7), such as upper maxilla-posterior region (20). The porous surface seems to be better fixed to bone, offering promising alternatives (7).

The scaffolds with a porous surface and dense nucleus, manufactured by powder metallurgy, combined important aspects such as greater compressive strength, efficient space for bone proliferation and improvement of the elastic modulus of titanium.

\section{References}

1. Brånemark PI. Osseointegration and its experimental background. J Prosthet Dent. 1983;50:399-410.

2. Kasemo B, Lausmaa J. Biomaterial and implant surfaces: a surface science approach. Int J Oral Maxillofac Implants. 1988;3:247-59.

3. Rak ZS, Walter J. Porous titanium foil by tape casting technique. J Mater process Tech 2005;175:358-63.

4. Spoerke ED, Murray NG, Li H, Brinson LC, Dunand DC, Stupp SI. A bioactive titanium foam scaffold for bone repair. Acta Biomater. 2005;1:523-33.

5. Cano J, Campo J, Vaquero JJ, Martínez González JM, Bascones A. High resolution image in bone biology II. Review of the literature. Med Oral Patol Oral Cir Bucal. 2008;13:E31-5.

6. Brentel AS, Vasconcellos LM, Oliveira MV, Graça ML, Vasconcellos LG, Cairo CA, et al. Histomorphometric analysis of pure titanium implants with porous surface versus rough surface. J Appl Oral Sci. 2006;14:213-8.

7. Vasconcellos LM, Oliveira MV, Graça ML, Vasconcellos LG, Cairo CA, Carvalho YR. Design of dental implants, influence on the osteogenesis and fixation. J Mater Sci Mater Med. 2008;19:2851-7.

8. Vasconcellos LMR, Oliveira MV, Graça MLA, Vasconcellos LGO, Cairo CAA, Carvalho YRC. Porous titanium scaffolds produced by powder metallurgy for biomedical applications. Materials Research 2008; 11:275-80.

9. Likibi F, Assad M, Coillard C, Chabot G, Rivard CH. Bone integration and apposition of porous and non porous metallic orthopaedic biomaterials. Ann Chir. 2005;130:235-41.

10. Wen CE, Yamada Y, Shimojima K, Chino Y, Asahina T, Mabuchi M. Processing and mechanical properties of autogenous titanium implant materials. J Mater Sci Mater Med. 2002;13:397-401.

11. Li JP, Li SH, Van Blitterswijk CA, De Groot K. Cancellous bone from porous Ti6Al4V by multiple coating technique. J Mater Sci Mater Med. 2006;17:179-85.

12. Svehla M, Morberg P, Zicat B, Bruce W, Sonnabend D, Walsh WR. Morphometric and mechanical evaluation of titanium implant integration: comparison of five surface structures. J Biomed Mater Res. 2000;51:15-22.

13. Zinger O, Zhao G, Schwartz Z, Simpson J, Wieland M, Landolt $D$, et al. Differential regulation of osteoblasts by substrate microstructural features. Biomaterials. 2005;26:1837-47.

14. Ryan G, Pandit A, Apatsidis DP. Fabrication methods of porous metals for use in orthopaedic applications. Biomaterials. 2006;27:2651-70.

15. Sepulveda P, Bressiani AH, Bressiani JC, Meseguer L, König B $\mathrm{Jr}$. In vivo evaluation of hydroxyapatite foams. J Biomed Mater Res. 2002;62:587-92.

16. Thelen S, Barthelat F, Brinson LC. Mechanics considerations for microporous titanium as an orthopedic implant material. J Biomed Mater Res A. 2004;69:601-10.

17. Torres FG, Nazhat SN, Sheikh SH, Fadzullah MD, Maquet V, Boccaccini AR. Mechanical properties and bioactivity of porous PLGA/TiO2 nanoparticle-filled composites for tissue engineering scaffolds. Comp Science and Techn. 2007;67:1139-47. 
18. Hing KA, Best SM, Tanner KE, Bonfield W, Revell PA. Quantification of bone ingrowth within bone-derived porous hydroxyapatite implants of varying density. J Mater Sci Mater Med. 1999;10:66370.

19. Cook SD, Rust-Dawicki AM. In vivo evaluation of a CSTi dental implant: a healing time course study. J Oral Implantol. 1995;21:8290.

20. Boronat López A, Balaguer Martínez J, Lamas Pelayo J, Carrillo García C, Peñarrocha Diago M. Resonance frequency analysis of dental implant stability during the healing period. Med Oral Patol Oral Cir Bucal. 2008;13:E244-7. 